\title{
Tensor Analysis-based Nonlocal Magnetic Resonance Image Reconstruction with Compressed Sensing
}

\author{
Qidi $\mathrm{Wu}^{\mathrm{a}}{ }^{\mathrm{*}}$ and Yibing $\mathrm{Li}^{\mathrm{b}}$ \\ ${ }^{a}$ Harbin Engineering University, Harbin, 150001, China \\ ${ }^{b}$ Daqing Vocational College, Daqing, 163255, China
}

\begin{abstract}
Compressed sensing (CS) is a novel and important technique in MRI reconstruction, which can be used to reconstruct magnetic resonance images with few sampled data while simultaneously speeding up imaging. The conventional CS-based MRI is implemented from a global view, which leads to some disadvantages: it not only loses many local structures but also fails to preserve detail information. To obtain better reconstruction quality, we propose a novel CS-based reconstruction model, which is incorporated with nonlocal technology to allow for the preservation of extra details. The proposed model groups similar patches within the nonlocal area and stacks them to form a 3D array unit. Then, to process the array in a realistic 3D manner, a tensor-based sparsity constraint is developed as the regularization on the reconstructed image. Experimental results show that the performance of the proposed method is superior to those of conventional methods.
\end{abstract}

Keywords: MRI; compressed sensing; sparse representation; tensor; nonlocal technology

(Submitted on May 20, 2018; Revised on June 29, 2018; Accepted on July 27, 2018)

(C) 2018 Totem Publisher, Inc. All rights reserved.

\section{Introduction}

Felix Block and Eduard Purcell independently discovered the magnetic resonance (MR) phenomenon in 1946. Since then, MR has been developed and applied in many different ways, and it has also been used in physical and chemical aspects of molecular analyses and reaction processes [1]. In 1967, Jasper Johns obtained an MR signal from a living animal for the first time and applied it to human MRI-related research [2]. Raymond Damadian discovered in 1971 that the MRI relaxation times of tissues and tumors are different, and this technique has played a large role in the detection of diseases. In 1975, Richard Ernst first proposed the use of frequency and phase coding techniques in the imaging process, which laid the foundation for the development of the modern MRI [3]. After decades of development, MRI imaging technology has become more sophisticated and has been used in many fields.

MRI is slow because the acquisition process must be calibrated based on the patient and based on very strict Nyquist sampling requirements. Subsequent reconstructions are done using a Fast Fourier Transform (FFT). In 2004, with the development of information theory and sparse theory, E. J. Candes, D. L. Donoho, and other scientists proposed the concept of Compressed Sensing (CS) [4] in the article on information and approximation theory and pointed out that it is not satisfied. In the case of Shannon Theorem, sampling below the Nyquist sampling frequency can restore the original signal from a small amount of measured data. The CS method developed as a signal processing and mathematics-related field can reduce the amount of data collected and save time. Because MRI as a basic medical imaging method is slow in data acquisition, CS is applied to it. MRI can significantly reduce the scan time, which will undoubtedly play a revolutionary role in patient care. In 2007, Lustig and other scholars at Stanford University used CS for MRI for the first time to achieve rapid MR imaging [5]. In recent years, CS-MRI research has been increasingly favored by scholars in related fields. Many scholars have devoted themselves to studying the more sparse transformation of MRI images, the optimal K-space sampling trajectory, and the more effective CS reconstruction algorithm.

* Corresponding author.

E-mail address: iseeklin@163.com 
CS was first used in signal processing related fields, which broke through the Nyquist sampling frequency limit, making it possible to capture high-resolution signals. In recent years, people have sought to improve the imaging speed of MRI. The introduction of the CS method has greatly changed traditional MRI imaging methods. CS can reduce the number of samples required, and at the same time it can improve the spatial and temporal reconstruction of images. It can reduce scanning time and improve imaging speed without the need to improve the gradient performance of MRI equipment [6].

In light of the advantages of CS-based methods, we propose a novel magnetic resonance image reconstruction method based on CS, which utilizes nonlocal redundant structural information and establishes a tensor-based reconstruction algorithm to further improve performance.

The rest of this paper is organized as follows. In Section 2, some previous classical works are discussed. Section 3 presents our proposed framework and its algorithm. The experiments are implemented in Section 3. Section 4 summarizes this paper.

\section{Previous Works}

In MRI imaging, CS-MRI related research has received more and more attention and has potential significance in accelerating imaging [7]. Motivated by successful achievements of regularization-based techniques in image reconstruction issues [8-10], many regularization-based methods have been studied in recent years. Michael Lustig et al. applied CS to MRI for the first time, established the entire model, and proposed the use of the non-linear conjugate gradient algorithm to solve this optimization problem. Assuming there are $\mathrm{m}$ samples and the original signal is $\mathrm{x}$, if the actual number of samples is $\mathrm{p}<\mathrm{m}$, then the matrix form is as follows:

$$
A x=b
$$

Where $A \in C^{p \times n}$ is the measurement matrix, $x \in C^{n}$ is the original signal, $b \in C^{p}$ is the measured value, and $\mathrm{x}$ is the $2 \mathrm{D}$ image, assuming that $\mathrm{x}$ is a column vector and $n=n_{1} \times n_{2}$. Providing $\mathrm{x}$ is sparse itself, then the solution of Equation (1) is the following formula:

$$
\arg \min _{x}\left\{\|\mathrm{x}\|_{0}\right\} \quad \text { subject to } A x=b
$$

Or, when considering the noise:

$$
\arg \min _{x}\left\{\|\mathrm{x}\|_{0}\right\} \quad \text { subject to }\|A x-b\|_{2}^{2}<\epsilon
$$

It is difficult to directly solve Equation (3). In [8], it is indicated that when using the regularized term $\ell_{1}$ norm to replace $\ell_{0}$ norm $\|x\|_{0}$, Equations (1-3) become:

$$
\arg \min _{x}\left\{\|\mathrm{x}\|_{1}\right\} \quad \text { subject to }\|A x-b\|_{2}^{2}<\epsilon
$$

In addition, [11] pointed out that the full variational regularization term TV $\bullet$ can make the reconstructed image more of a sparse representation and introduce this term into the CS reconstruction model [12]. For a specific $x \in C^{n_{1} \times n_{2}}$, this is defined as:

$$
\|\mathrm{x}\|_{T V}=\sum_{i=1}^{n_{1}-1} \sum_{j=1}^{n_{2}-1} \sqrt{\left|x_{i, j}-x_{i+1, j}\right|^{2}+\left|x_{i, j}-x_{i, j+1}\right|^{2}}+\sum_{j=1}^{n_{1}-1}\left|x_{i, n_{2}}-x_{i+1, n_{2}}\right|+\sum_{j=1}^{n_{2}-1}\left|x_{n_{1}, j}-x_{n_{1}, j+1}\right|
$$

In general, if $\mathrm{x}$ is not sparse in the time domain, then the sparse transform $\Psi$ needs to be introduced. The optimization problem of the CS model containing the sparse transform and the full variational regularization term becomes:

$$
\arg \min _{x}\left\{\theta_{1}\|\Psi \mathrm{x}\|_{1}+\theta_{2}\|x\|_{T V}\right\} \quad \text { subject to }\|A x-b\|_{2}^{2}<\epsilon
$$

Where the parameters $\theta_{1}, \theta_{2}>0$. Introducing the Lagrangian multiplier $\lambda$, Equation (6) becomes: 


$$
\arg \min _{x}\left\{\frac{1}{2}\|A x-b\|_{2}^{2}+\alpha\|x\|_{T V}+\beta\|\Psi x\|_{1}\right\}
$$

Where $\alpha$ and $\beta$ are regularization parameters and $\alpha=\theta_{2} / 2 \lambda, \beta=\theta_{1} / 2 \lambda$. Equation (7) demonstrates the mathematical model of the entire reconstruction of CS-MRI.

Xiaobo Qu et al. proposes a method for sparsifying an image using the similarity of image patches [13]. Consider the data acquisition model:

$$
y=F_{U} x+\varepsilon
$$

Where $x \in C^{N}$ is the discretized image to be reconstructed; $y \in C^{M}$ is the acquired k-space data; $F_{U} \in C^{M \times N}(M<N)$ is the under sampled Fourier transform operator, which directly relies on the sampling scheme [14]; and $\varepsilon \in C^{M}$ is the noise vector. A typical CS-MRI reconstruction attempts to solve the following problem [15]:

$$
\hat{x}=\arg \min _{x}\left\{\left\|\psi^{T} x\right\|_{1}+\frac{\lambda}{2}\left\|y-F_{U} x\right\|_{2}^{2}\right\}
$$

Where $\psi^{T}$ is a sparsifying transform of $x$; the $\|\cdot\|_{1}$ stands for the $\ell_{1}$ norm, which promotes the sparsity of $\psi^{T} x$; and the $\|\cdot\|_{2}$ stands for the $\ell_{2}$ norm, which enforces the fidelity of the reconstruction to the measured $\mathrm{k}$-space data. The regularization parameter $\lambda$ decides the tradeoff between the sparsity and the data fidelity.

Although CS-MRI is promising for reconstruction from undersampled k-space data, the usage of a pre-defined sparsifying transform sometimes leads to an insufficient sparse representation for certain types of images, thus resulting in artifacts in the reconstruction. For example, the commonly used 2D separable wavelet fails in capturing smooth contours [16-17], and finite differences may introduce staircase artifacts [18-19] at high undersampling factors. We aim to construct a better sparsifying transform by making use of the nonlocal similarity of image patches to achieve higher undersampling factors than the conventional CS-MRI.

\section{The Proposed Method}

Though some conventional methods with nonlocal grouping technology have been proposed to address the MRI reconstruction problem, the realistic 3D transformation has not been implemented on the nonlocal patches array. In general, the so-called 3D transformation is implemented with an alternative 2D and 1D transformation respectively, which cannot effectively capture the 3D similar structure in these nonlocal patches. Therefore, we propose to introduce the tensor-based spare representation into the conventional model and establish a meaningful reconstruction method for MRI based on 3D nonlocal grouping technology.

\subsection{Tensor-based Sparsity Decomposition}

Firstly, we illustrate the notations used in this paper. The term tensor refers to a multidimensional array, also known as a multi-way or multi-mode array. We denote scalars with lower-case letters $(a, b, \cdots)$, matrices with capital letters $(M, H, \cdots)$, and tensors with bold-face capital letters $(\boldsymbol{C}, \boldsymbol{T}, \cdots)$. The $i^{\text {th }}$ unfolding of tensor $\boldsymbol{T}$ is denoted by $T_{(i)}$.

According [20-21], the high-order singular value decomposition perceives that each $d^{\text {th }}$ order tensor $\boldsymbol{T} \in \boldsymbol{R}^{n_{1} \times n_{2} \times \cdots \times x_{d} n_{d}}$ can be presented by a tensor $\boldsymbol{C}$ and $d^{\text {th }}$ matrices, as follows:

$$
\boldsymbol{T}=\boldsymbol{C} \times{ }_{1} H_{1} \times{ }_{2} H_{2} \times \cdots \times{ }_{d} H_{d}
$$


Where $H_{i} \in \boldsymbol{R}^{n_{i} \times r_{i}}$ is an orthogonal matrix that captures structural information of $T_{(i)}$ with $r_{i}=\operatorname{rank}\left(T_{(i)}\right), i=1,2, \cdots, d$, and $\boldsymbol{S} \in \boldsymbol{R}^{r_{1} \times r_{2} \times \cdots r_{d}}$ is the so-called core tensor which describes the contributions of $\boldsymbol{T}$. The operator $\times_{i}$ denotes the mode- $i$ product of the tensor with a matrix. It is noted that if $U \in \boldsymbol{R}^{r_{i} \times n_{i}}$, then $\boldsymbol{T} \times{ }_{i} H \in \boldsymbol{R}^{n_{1} \times \ldots i_{i} \times \cdots n_{d}}$.

Furthermore, the tensor S satisfies the following properties:

(1) $\|\boldsymbol{T}\|_{F}=\|\boldsymbol{C}\|_{F}$, where the Frobenius norm is defined as $\|\boldsymbol{T}\|_{F}=\sqrt{\sum_{i_{1}, i_{2}, \ldots, i_{d}}\left(t_{i_{1}, i_{2}, \ldots, i_{d}}\right)^{2}}$.

(2) The sub-tensors should be ordered decreasingly by their Frobenius norms as the following form:

$$
\left\|\boldsymbol{C}_{i_{k=1}}\right\|_{F} \geq\left\|\boldsymbol{C}_{i_{k=2}}\right\|_{F} \geq \cdots\left\|\boldsymbol{C}_{i_{k=d}}\right\|_{F} \geq 0
$$

(3) $C_{i_{k}}$ are orthogonal to each other, which means $\left\langle C_{i_{p}}, C_{i_{q}}\right\rangle=0$ if $p \neq q$.

With the above notation, we propose a tensor-based soft-thresholding shrinkage (TSTSA) algorithm to solve the following sparsity problem for 3D arrays:

$$
\min _{Q}\left\|Q-T_{3 D}(X)\right\|_{F}^{2}+\theta\|W \otimes Q\|_{1}
$$

Where $T_{3 D}(X)$ presents a tensor-based 3D transformation for $X$, and $Q$ denotes the tensor-based sparse component of $T_{3 D}(X) . \otimes$ denotes the Hadamard production. In addition, to improve the performance, we introduce the weighted constraint matrix $W$. The coefficient in $Q$ presents the correlation between the signal and transformation basis unit. Therefore, to further enforce the correlation, we take the inner-product of the signal and transformation basis as an element of $W$, such as $W_{i j}=\left\langle\operatorname{vec}\left(X_{i}\right)\right.$,vec $\left.\left(H_{j}\right)\right\rangle$.

To solve the model in Equation (3), high-order SVD is first performed on the 3D array $X$. Secondly, the tensor-based soft-thresholding is applied. With an initial observation $X$, we can implement the high-order SVD as follows:

$$
T_{3 D}\left(X^{k-1}\right)=\mathbf{C}_{X} \times_{1} H_{1} \times_{2} H_{2} \ldots \times_{d} H_{d}
$$

Then, the $k^{\text {th }}$ approximate is obtained by:

$$
Q^{k-1}=S_{\eta}\left(\mathbf{C}_{X}\right) \times_{1} H_{1} \times_{2} H_{2} \ldots \times_{d} H_{d} Q^{k-1}=S_{\eta}\left(\mathbf{C}_{X}\right) \times_{1} H_{1} \times_{2} H_{2} \ldots \times_{d} H_{d}
$$

Where $S_{\eta}\left(\mathbf{C}_{X}\right)$ is the soft-thresholding shrinkage function, as follows:

$$
S_{\eta}(\mathbf{C})=\left\{\begin{array}{cl}
\mathbf{C}_{i_{1}, i_{2}, \ldots, i_{d}}-\operatorname{sgn}\left(\mathbf{C}_{i_{1}, i_{2}, \ldots, i_{d}}\right) \eta, & \text { if }\left|\mathbf{C}_{i_{1}, i_{2}, \ldots, i_{d}}\right|>\eta \\
0, & \text { otherwise }
\end{array}\right.
$$

\subsection{The Reconstruction Model}

For a given image $x \in C^{N}$, we first decompose it into some patches with fixed size $L \times L$. Let $R_{i}$ be the patch extraction operator, and the $i^{\text {th }}$ patch $x_{i} \in C^{L^{2}}$ can be presented as $x_{i}=R_{i} x$. Then, a group with $n_{i}$ patches, which are similar to $x_{i}$, is denoted as $U_{n_{i}} x_{i}$, where $n_{i}=\left\{i_{1}, \cdots, i_{K}\right\}$. It is used to save the index of similar patches. With the above definition, a groupbased nonlocal extractor is given as follows: 


$$
T_{i}=U_{n_{i}} R_{i}
$$

With Equation (16), we can obtain a patch group $G_{i}$ of $x_{i}$ as:

$$
G_{i}=T_{i} x
$$

For practical applications, we cannot obtain a referenced image to determine the location of similar patches. Therefore, in this paper, we follow the method in literature [14] to first generate a guided image. Then, we take the guided image to give the indexes of similar patches. The guided image can be obtained with any reconstruction method, such as the methods given in section 2. In our proposed method, we simply use the model in Equation (9) to obtain the guided image.

Then, the following Euclidean distance is used to search the similar patches for a known patch as:

$$
d\left(x_{i}, \tilde{x}_{i}\right)=\left\|x_{i}-\tilde{x}_{i}\right\|_{2}
$$

The operation in Equation (18) is similar to the so-called block matching in [22]. Furthermore, the search region can be as large as the entire image, so the similar patches are not limited to a local region but located at a nonlocal area, which will be advantageous in improving structure recovery.

By stacking the similar patches of group $G_{i}$ to a $3 \mathrm{D}$ array with the operator $S$, we propose the following reconstruction model:

$$
\min _{\mathbf{x}} \sum_{j=1}^{J}\left\|W \otimes S\left(G_{j}\right)\right\|_{1}+\frac{\lambda}{2}\left\|\mathbf{y}-\mathbf{F}_{\mathbf{U}} \mathbf{x}\right\|_{2}^{2}
$$

Where $x \in C^{N}$ is the original image to be reconstructed, $y \in C^{M}$ is the undersampled k-space data, and $F_{U} \in C^{M \times N}(M<N)$ denotes the Fourier transform with the undersampled mask $U$. W is the weighted matrix to give some extra constraint on the correlation between the patch in $G_{j}$ and the transformation basis.

Nevertheless, different from the conventional 3D process, we propose to take the first term in Equation (19) as a tensor and apply a tensor-based soft-thresholding method to obtain the approximate solution. To this end, we first introduce an auxiliary variable $M_{i}$ into the proposed model as follows:

$$
\begin{gathered}
\min _{\mathbf{x}} \sum_{j=1}^{J}\left\|W \otimes \phi\left(S\left(G_{j}\right)\right)\right\|_{1}+\lambda\left\|\mathbf{y}-\mathbf{F}_{\mathbf{U}} \mathbf{x}\right\|_{2}^{2} \\
\text { s.t } W \otimes \phi\left(S\left(G_{j}\right)\right)=M_{j}, \forall j
\end{gathered}
$$

Where $\phi$ is a tensor-based sparsity transform.

Based on the splitting technique, we can first transform Equation (20) to the unconstraint form as follows:

$$
\min _{x, M} \sum_{j=1}^{J}\left\|M_{i}\right\|_{1}+\eta\left\|M_{i}-W \otimes \phi\left(S\left(T_{i} \mathbf{x}\right)\right)\right\|_{2}^{2}+\lambda\left\|\mathbf{y}-\mathbf{F}_{\mathbf{U}} \mathbf{x}\right\|_{2}^{2}
$$

Then, to split the unconstraint form into the following two subproblems:

$$
\hat{M}_{i}=\arg \min \left\|M_{i}\right\|_{1}+\eta\left\|M_{i}-W \otimes \phi\left(S\left(T_{i} \mathbf{x}\right)\right)\right\|_{2}^{2}
$$




$$
\hat{\mathbf{x}}=\arg \min _{x} \sum_{j=1}^{J}\left(\eta\left\|M_{i}-W \otimes \phi\left(S\left(T_{i} \mathbf{x}\right)\right)\right\|_{2}^{2}\right)+\lambda\left\|\mathbf{y}-\mathbf{F}_{\mathbf{U}} \mathbf{x}\right\|_{2}^{2}
$$

Equation (22) can be solved using the proposed tensor-based sparsity decomposition method in section 3.1. As for $W$, we simply compute it with the result of the last iteration in our method.

Equation (23) is a quadratic subproblem for variables. By forcing its derivative to be zero, we can obtain the solution using the conjugate gradient method.

The synthesis algorithm can be described in the following table.

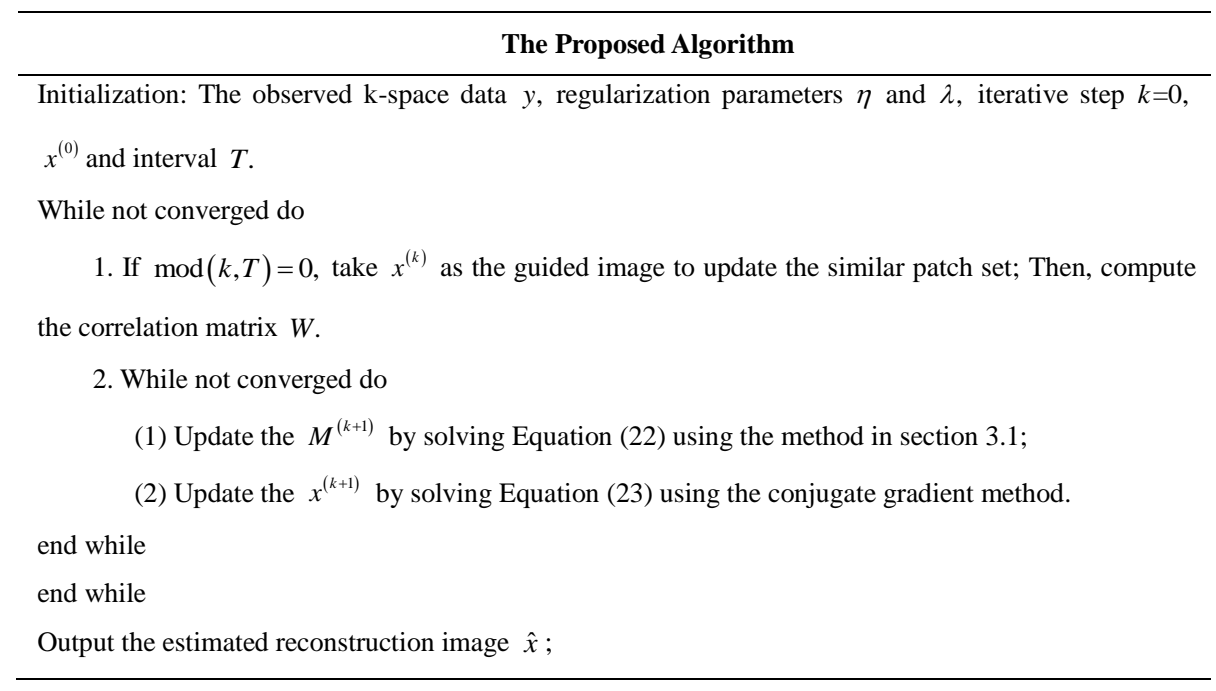

\section{Experimental Results and Analysis}

In this section, we conduct experiments on the $\mathrm{k}$-space data to reconstruct the magnetic resonance image. The comparison method includes the CS-based reconstruction with zero-filled low-frequency component (CSZFLF), conventional CS-based model(CCS) in [5], CS-based model via total variation (CSTV) [23]. and the proposed method. The parameters of the comparison method are tuned according to the original literatures. For the proposed method, the patch size $L$ and the number of similar patches are set as 7 and 10, respectively. The radius of the search region is 30 . The maximum value of the iterative number is set as 200. The sampling rate ranges from 0.2 to 0.8 in the density Cartesian sampling way. The test images are shown in Figure 1. The reconstruction results of different test images with rate $=0.6$ are shown in Figures 2 and 3.

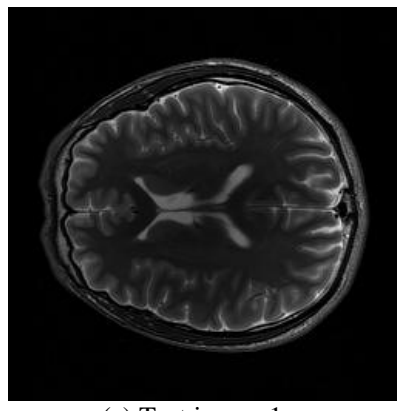

(a) Test image 1

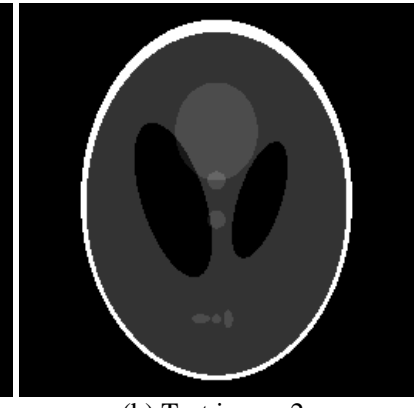

(b) Test image 2

Figure 1. Test Images

To evaluate the reconstruction performance, we adopt the relative error as an indicator, which is used to measure the quality compared to the fully sampled reconstructed image. The errors are depicted in Figure 4 under different undersampling rates. The definition of the relative error is shown as follows: 


$$
R L N E=\frac{\left\|\hat{x}-x_{F}\right\|_{2}}{\|\hat{x}\|_{2}}
$$

Where $\hat{x}$ is the reconstruction image and $x_{F}$ is the fully sampled image.
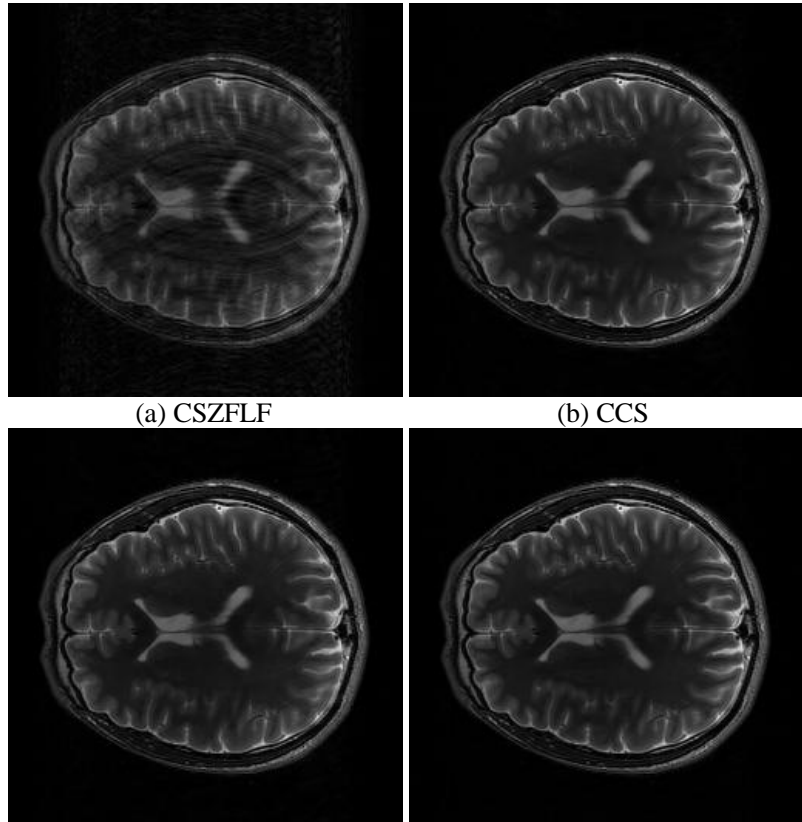

(c) CSTV

(d) Proposed

Figure 2. The reconstruction results of test image 1
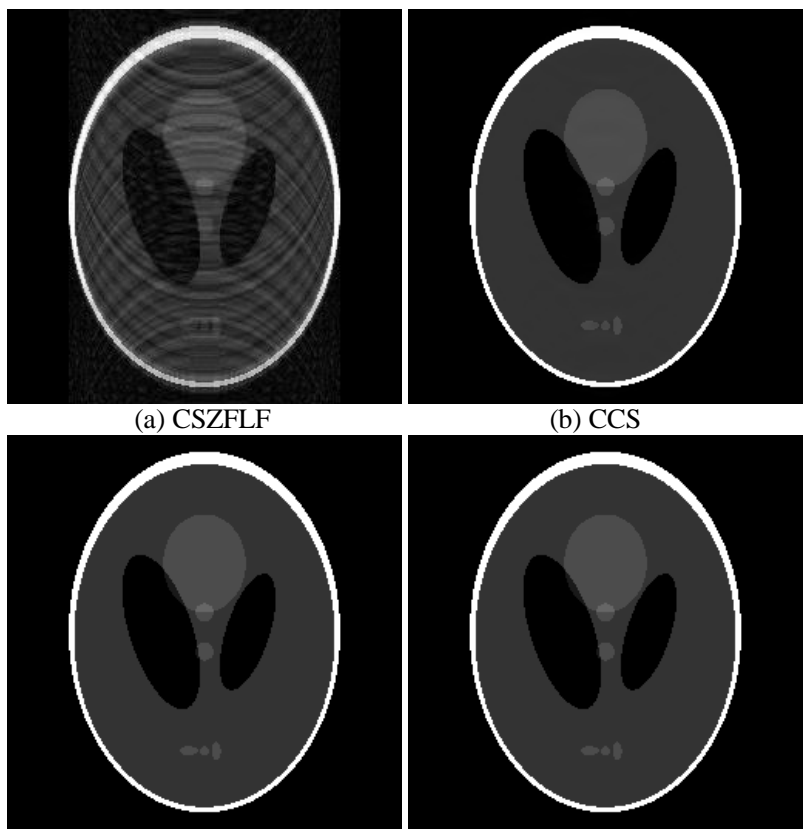

(b) CCS

(c) CSTV

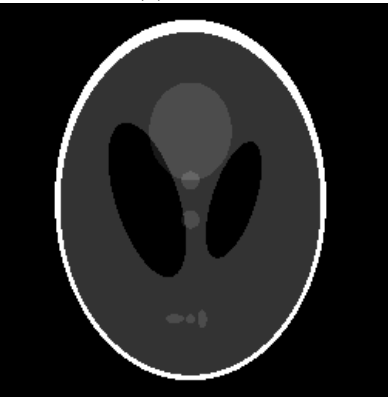

(d) Proposed

Figure 3. The reconstruction results of test image 2

From the visual results and objective indicators, we can see that the proposed method outperforms the conventional MRI reconstruction methods in both the visual quality and the objective evaluation indicator. 


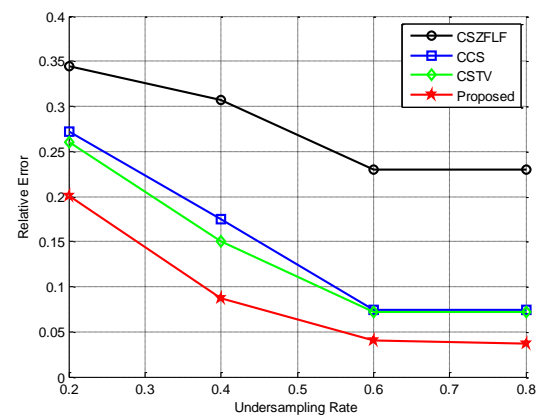

(a) Test image 1

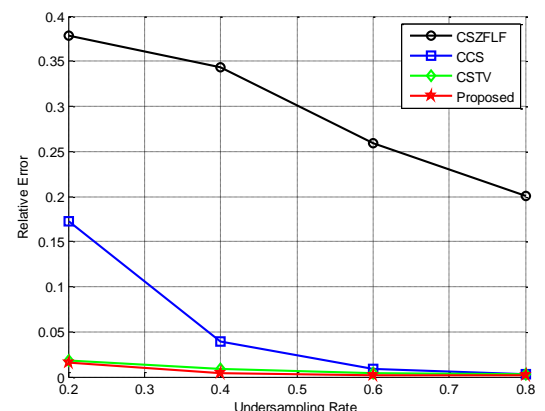

(b) Test image 2

Figure 4. Relative error of different methods

\section{Conclusion}

In this paper, a CS-based nonlocal tensor method is presented to address the MRI reconstruction problem. Nonlocal patches are grouped to form a 3D tensor matrix, which is used to better preserve the details existing in the original image. Then, the tensor-based weighted sparsity constraint is introduced to force the above 3D stacked matrix. To further improve the performance, the correlation matrix is also introduced to enforce the sparsity coefficient. Finally, the reconstruction image is computed by an iterative algorithm. The effectiveness and efficiency of the proposed method are verified through the comparison experiments.

\section{References}

1. R. W. Brown, Y. C. N. Cheng, E. M. Haacke, M. R. Thompson, R. Venkatesan, "Magnetic Resonance Imaging: Physical Principles and Sequence Design," 2nd Edition, J. Wiley \& Sons, 2013

2. B. K. Das, "Basic Principles of MR Imaging," Springer India, 2015

3. R. R. Ernst, “NMR Fourier Zeugmatography,” Journal of Magnetic Resonance, Vol. 213, pp. 510-512, 2011

4. E. J. Candes, M. B. Wakin, “An Introduction To Compressive Sampling,” IEEE Signal Process Magazine, Vol. 12, pp. 21-30, 2017

5. M. Lustig and D. J. Donoho, "Sparse MRI: The Application of Compressed Sensing for Rapid MR Imaging," Magnetic Resonance in Medicine, Vol. 58, pp. 1182-1195, 1999

6. J. Trzasko, C. Haider, and A. Manduca, "Practical Nonconvex Compressive Sensing Reconstruction of Highly-accelerated 3D Parallel MR Angiograms," in Proceedings of IEEE International Conference on Symposium on Biomedical Imaging: from Nano to Macro, pp. 274-277, 2009

7. K. P. Pruessmann, M. Weiger, M. B. Scheidegger, and P. Boesiger, "SENSE: Sensitivity Encoding for Fast MRI," Magnetic Resonance in Medicine, Vol. 42, pp. 952-962, 1999

8. Manisha, G. Shirividya, S. H. Bharati, et al., "Performance Analysis of CS-MRI Reconstruction using Particle Swarm Optimization for Different Sampling Patterns," in Proceedings of International Conference on Recent Trends in Electronics, Information \& Communication Technology, pp. 205-208, 2017

9. A. Li, D. Chen, K. Lin, and G. Sun, "Hyperspectral Image Denoising with Composite Regularization Models," Journal of Sensors, pp. 1-10, 2016

10. A. Li, D. Chen, K. Lin, and G. Sun, "Sparse Representation-based Image Restoration via Supervised Coding," Optical Review, Vol. 23, No. 5, pp. 776-783, 2016

11. A. Li, D. Chen, Z. Wu, G. Sun, and K. Lin, "Self-supervised Sparse Coding Scheme for Image Classification based on Low Rank Representation," PLOS ONE, Vol. 13, No. 6, pp. 1-15, 2018

12. G. Shrividya, S. H. Bharathi, et al., "Performance Analysis of CS-MRI Reconstruction using Particle Swarm Optimization for Different Sampling Patterns," Recent Trends in Electronics, Information \& Communication Technology (RTEICT), pp. 205-208, 2017

13. S. Geethanath, R. Reddy, A. S. Konar, S. Imam, and R. Sundaresan, "Compressed Sensing MRI: A Review," Crit Rev Biomed Eng, Vol. 41, pp. 183-204, 2013

14. X. Qu, Y. Hou, F. Lam, D. Guo, and J. Zhong, "Magnetic Resonance Image Reconstruction from Undersampled Measurements using A Patch-based Nonlocal Operator,” Medical Image Analysis, Vol. 18, pp. 843-856, 2014

15. M. Lustig, J. M. Santos, J. H. Lee, D. L. Donoho, and J. M. Pauly, “Application of "Compressed Sensing” for Rapid MR Imaging," Spars, Vol. 58, pp. 1182-1195, 2010 
16. S. M. Gho, Y. Nam, S. Y. Zho, E. Y. Kim, and D. H. Kim, “Three Dimension Double Inversion Recovery Gray Matter Imaging using Compressed Sensing," Magnetic Resonance Imaging, Vol. 28, pp. 1395-1402, 2010

17. X. Qu, W. Zhang, D. Guo, C. Cai, and S. Cai, "Iterative Thresholding Compressed Sensing MRI based on Contourlet Transform," Inverse Problems in Science \& Engineering, Vol. 18, pp. 737-758, 2010

18. F. Knoll, K. Bredies, T. Pock, and R. Stollberger, "Second Order Total Generalized Variation (TGV) for MRI," Magnetic Resonance in Medicine, Vol. 65, pp. 480-491, 2011

19. Y. Dong and J. Ji, "Compressive Sensing MRI with Laplacian Sparsifying Transform," Proceedings, Vol. 48, pp. 81-84, 2011

20. L. D. Lathauwer, B. D. Moor, and J. Vandewalle J, "A Multilinear Singular Value Decomposition," SIMA Journal on Matrix Analysis and Applications, Vol. 21, pp. 1253-1278, 2000

21. T. G. Kolda and B. W. Bader, "Tensor Decompositions and Applications," in Proceedings of IEEE Conference on Decision and Control, Vol. 641, pp. 640-645, 2004

22. K. Dabov, A. Foi, V. Katkovnik, and K. Egiazarian, "Image Denoising by Sparse 3-D Transform-Domain Collaborative Filtering," IEEE Transactions on Image Processing, Vol. 16, pp. 2080, 2007

23. L. Sun, Y. Huang, C. Cai, and X. Ding, "Compressed Sensing MRI using Total Variation Regularization with K-space Decomposition," in Proceedings of IEEE International Conference on Image Processing, pp. 3061-3065, 2017 